\title{
A Hardware-In-The-Loop Simulation Architecture for Integration of Smart Buildings and Distributed Energy Resources in Micro Grids
}

\author{
G. Costanzo, L. Ferrarini, G. Mantovani, \\ A. Krusteva, M. Georgiev, M. Antchev
}

\section{Introduction}

Energy resources like wind and solar have an intrinsic variability that can seriously affect the power grid stability especially in scenarios with high penetration of micro generation at the distribution level. Nonetheless, local generation can significantly reduce the carbon footprint of human activities and urban development. The increasing interest for green energy leads to the incentive to create the installation of small PV arrays in many European countries at the point that the stability of the distribution grid becomes a major issue because of over voltages and reversed power flows [1-7].

However, in the near future it is plausible to foresee an increase of distributed storage, provided by electric vehicles (EVs), and flexible consumption enabled by the demand-side management in buildings. Since the latter technology leads to higher demand flexibility and predictability, allowing peak shaving, valley filling, load shifting and realtime power balancing $[1,4]$, the integration and coordination of Demand-Side Resources (DSRs) at the distribution level is becoming of relevant interest.

This project consists of the following activities:

- Design of hardware-in-the-loop (HIL) simulation architecture.

- Design and test of Model Predictive Control schemes (MPC) for the integration of DSRs in micro-grids.

- Implementation and test of controllers on a HIL platform.

The purpose and contribution of this study consists in the design of a simple and flexible HIL simulation archi- tecture by using off-the-shelves products and technologies. As a proof of the concept, this paper presents HIL simulations for testing advanced control schemes for DSRs coordination in Micro Grids under Demand Response environment.

The rest of the paper is organized as follows: Section II presents the proposed architecture for HIL simulation. Section III presents the experimental setup and the preliminary tests for hardware operation. Section IV introduces the models of Demand-Side Resources (DSRs), while the control strategies are given in details in Section V. Section VI discusses the preliminary simulation results and the conclusions are presented in Section VII.

\section{Description of the Architecture}

The proposed architecture allows integrating off-theshelves devices in a HIL simulation setup within software programming. All the devices are installed and set up in compliance with manufacturer safety directives, while custom-made software performs supervisory control and data acquisition (SCADA) (figure 1).

The SCADA consists of several major elements: an OPC Server, an HMI (Human Machine Interface), an IEC6185 server and an XML server. The OPC and HMI collect data from the field devices and archive them in a database. The OPC server has two client drivers: one for the field devices

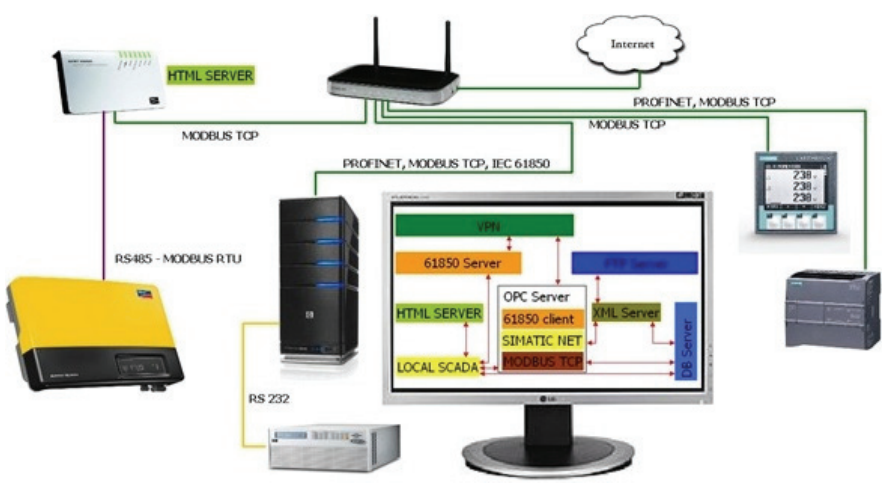

Figure 1. Schematics of the supervisory control and data acquisition system for HIL setup 


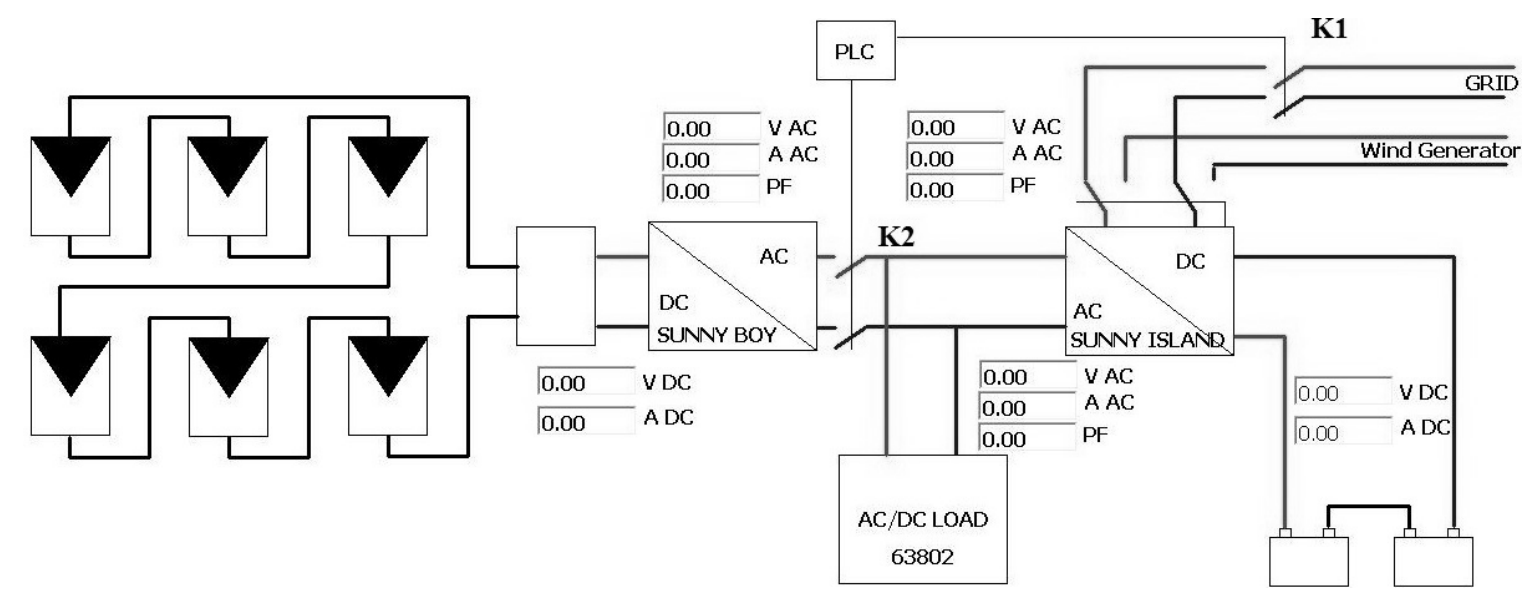

Figure 2. Test bench for installation at Technical University of Sofia - PEL (Power Electronics Laboratory)

protocol and one for exchanging data with the IEC61850 server. A software module (PROFINET to IEC61850 Gateway) exchanges data between the field devices and the IEC61850 server. All control and monitoring values are accessible through OPC and IEC61850 Servers. The HMI is used for local visualization and control of the system.

Since the electronic load used has ASCII protocol to transfer control commands, a scheme with XML files is exchanged for controlling it. The XML files consist of a list with commands to the load. The XML files are transferred through FTP server and the commands are downloaded to the electronic load by a software module (XML server). The feedback from the facility is also transferred in XML format. The archive values in $c s v$ format are accessible on demand at FTP server. Control signals to change the different facility regimes could be exchanged using both ways (through IEC6185 server or through XML server).

With this setup it is possible to perform HIL simulations with different components, such as controllers and DSRs models, implemented and running on computers placed elsewhere the installation and communication is kept, simply thanks to the information exchange via formatted text files.

\section{Experimental Setup}

The HIL simulation setup consists of:

- Six PV units Sharp NT175.

- One PV inverter SMA Sunny Boy 1200.

- One inverter SMA Sunny Island 2224 (grid-forming unit).

- Two batteries CSB GPL121000 (12V, 100Ah).

- One controllable load Chroma AC 63802.

- Windows-based computer with Internet connection.

The field measurements, such as currents and voltages, as well as control signals, such as controls for circuit breakers, are managed by Siemens PLC that communicates with the local SCADA. The controllable load communicates with the local SCADA via RS 232. Different experimental regimes are realized:

- Normal Operation Mode (grid and PV are connected) - NOM.
- Grid Operation Mode (without PV generation) - GOM.

- Island Operation Mode (islanded, with PV generation) - IOM.

- Batteries Operation Mode (islanded, without PV generation) - BOM.

As a preliminary test, BOM mode is investigated (figure 3).

The Sunny Island can operate in a certain P-Q range, outside which it enters a protection mode causing the micro grid to collapse.

The test shows the grid forming unit performances in maintaining the voltage and frequency under particular load conditions. The test is realized by varying the power factor between 0 and 1 at every value of active power, which is spanned between $0.2 \mathrm{~kW}$ and $1.6 \mathrm{~kW}$ at $0.2 \mathrm{~kW}$ steps (figure 3).

The following data is gathered for every regime:

- Voltage of the batteries through resistors with accuracy of $1 \%$.

- Current in or out of the batteries through LEM current transducer LA55-P.

The operation mode can be selected through a local SCADA or through XML command using the same XML scheme as in the load control system.

As an example, in the grid connected operation it is possible to remotely disconnect the Sunny Boy and measure the charge/discharge current and the voltage at the batteries. The level of discharge will depend on the grid voltage (figure 4).

The overall testing and control architecture is shown in figure 5. In this, you can select the setup operation modes and the HIL test of the control architectures based on MPC (Model Predictive Control) for building space heating under Demand Response conditions.

In figure 6 the communication architecture is given. Basically a Java-based gateway guarantees the synchronization between the field components and the simulation, and commands and data are exchanged through suitably formatted XML files. The communication layer developed in Java reads the simulation data coming from Matlab 

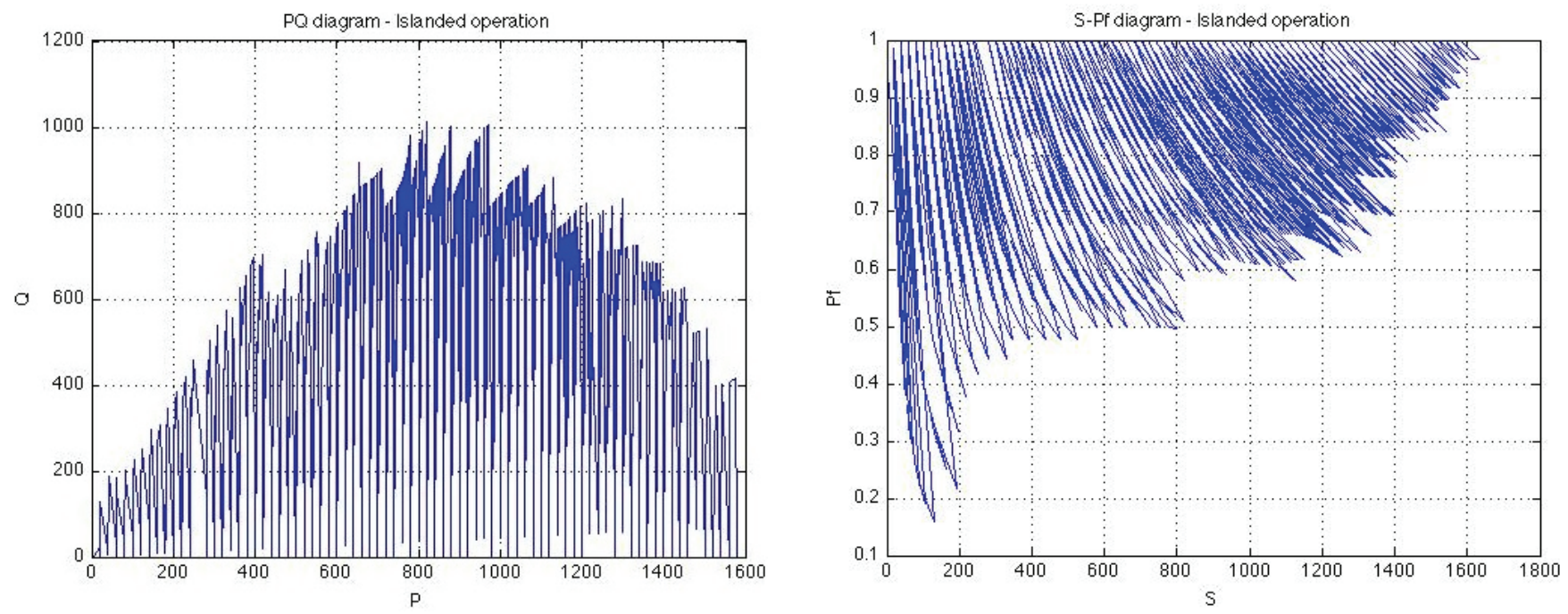

Figure 3. BOM mode: the micro grid is stable for PQ values in the blue area (figure $3 a$ ) and S-pf diagram $(b)$

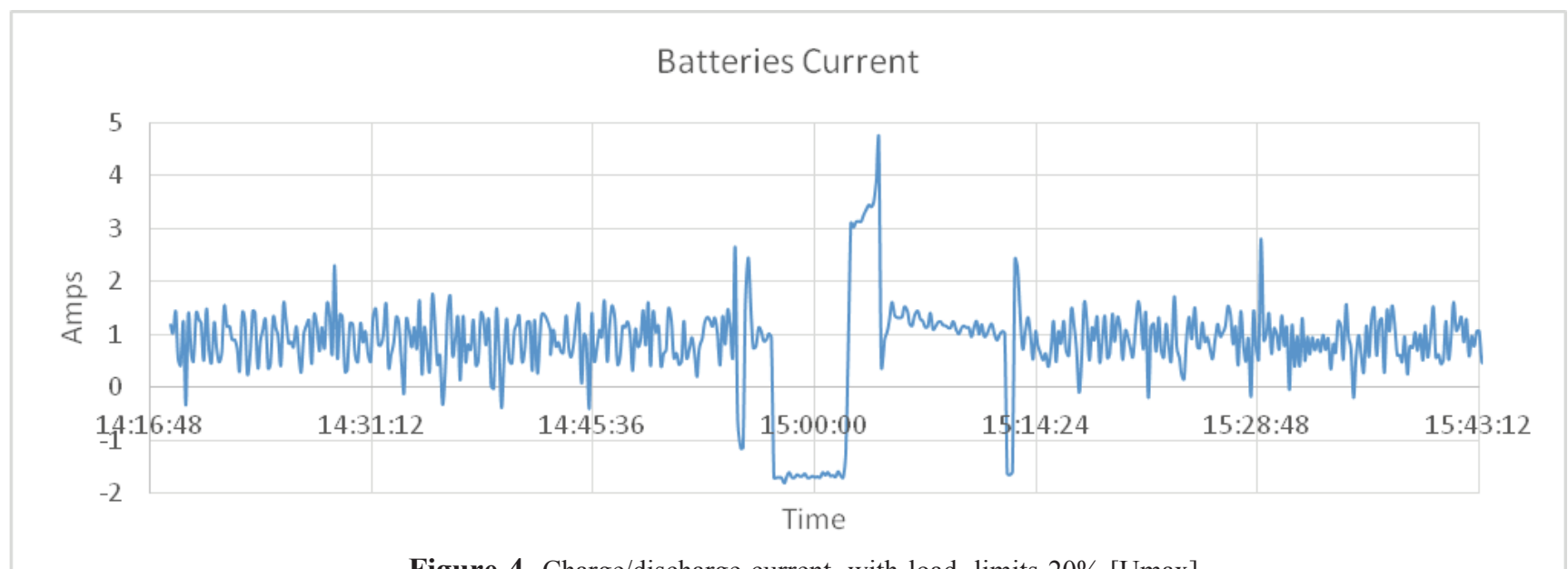

Figure 4. Charge/discharge current, with load, limits 20\% [Umax]

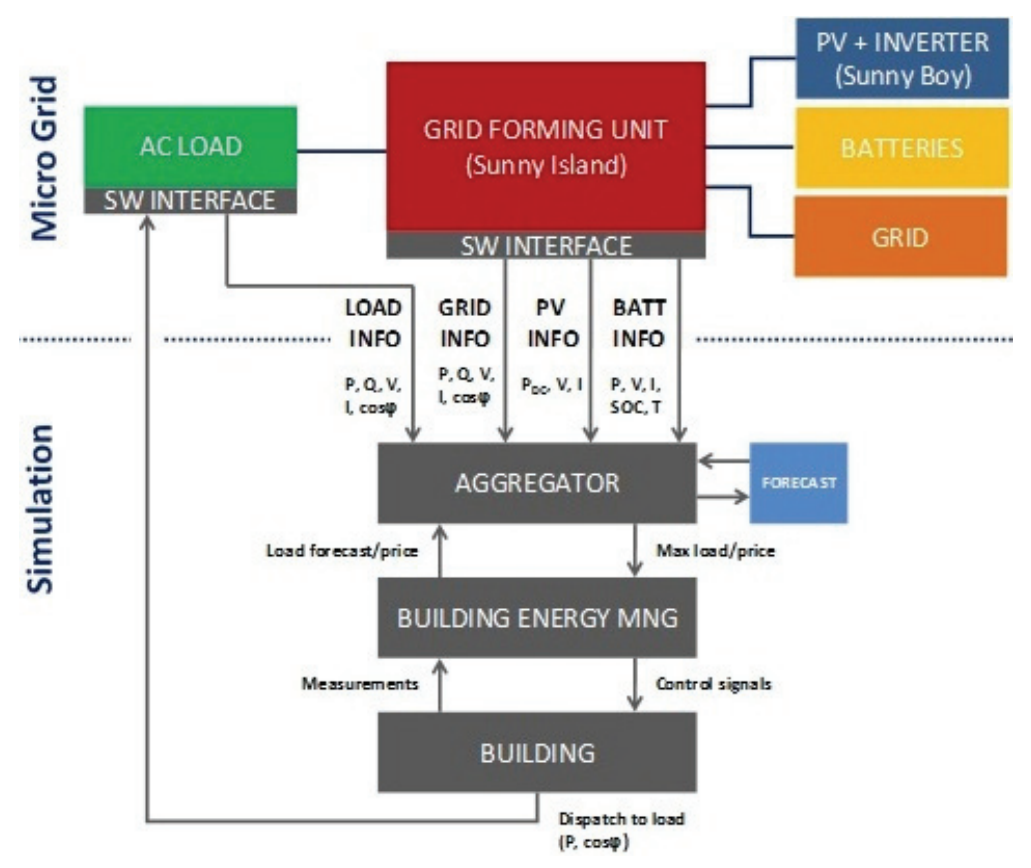

Figure 5. Block scheme of the hybrid system with a control loop 


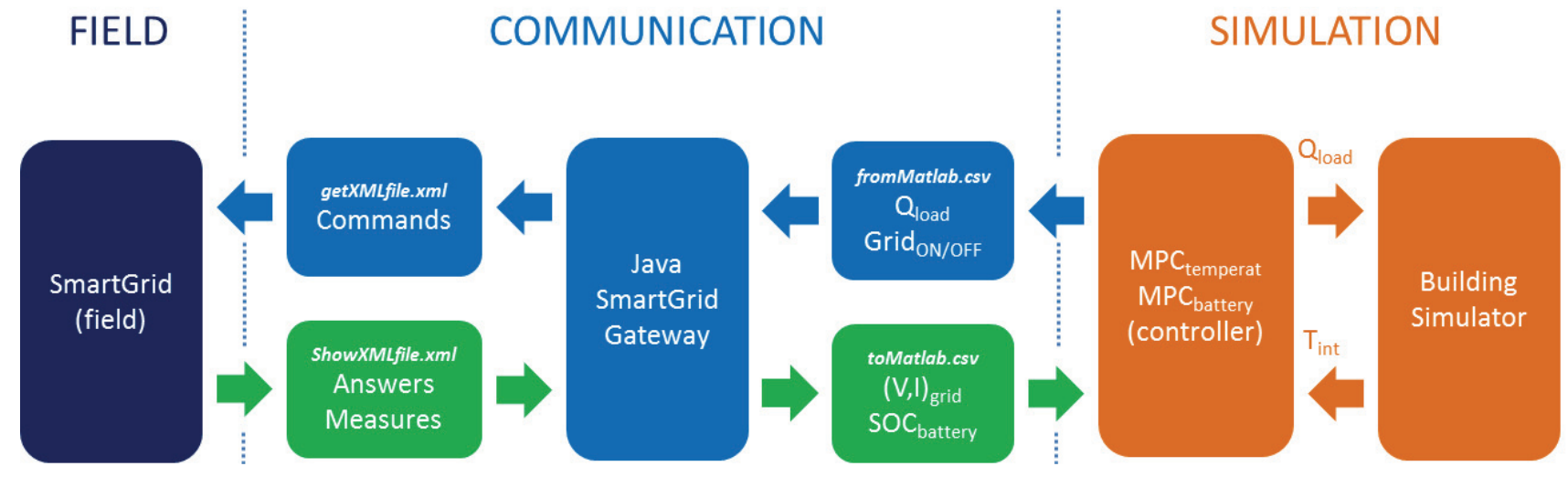

Figure 6. Overall communication architecture

(a building simulator and MPC controllers for optimal regulation of the building temperature and smart grid batteries) and sends it to the hardware setup commands for dispatching the load and set the islanded or grid connected modes (connects or disconnects the Sunny Island to the grid). Notice that in Grid ON mode, the batteries are kept charged from the Sunny Island, while in Grid OFF mode the batteries are used to balance the power absorbed by the AC load, which is controlled by the MPC algorithms) and the $\mathrm{PV}$ production.

\section{Models for DERs}

In the present section, the models for the main components present in the considered grid are briefly described.

\section{1) Building and Space Heating}

The building heat dynamics is modeled by the following transfer function:

$$
\text { (1) } \frac{T(s)}{U(s)}=\frac{0.1116}{16000 s+1} \text {. }
$$

As discussed in [6], the model is a simplified single volume model of a commercial building located in northern Italy (Gavirate, VA). It has thirty shops and consists of five floors. The building envelope is characterized by a layered and insulated wall. The roof is partially covered by a $7 \mathrm{~mm}$ glass cover. The centre is cooled in summer by two $1 \mathrm{MW}$ refrigeration units, and heated in winter by a combination of district heating and heat pumps. The installed building automation system (BAS) monitors the consumption data coming from all the electrical and thermal loads in the shopping center. The single volume model is a simplified model to show the main advantages of the control and demand response without the burden of too many equations, but the original model is a 5-volume stratified model which includes the model of the envelope, infiltrations, thermal plants, fan coils, internal gains due to people, solar radiation, and external temperature.

\section{2) Battery Model}

A detailed model of the electric storage units is pre- sented in [2]. For this application, however, it is reasonable to model the energy storage together with the power electronics in a single unit. Maintaining the state of charge (SOC) between $20 \%$ and $90 \%$, it is possible to preserve the battery lifetime and control the battery charge/discharge at a constant current mode. Therefore, the battery is modeled here as an integrator of rated efficiency. The characteristics of the storage units are depicted in Appendix I, while the model equation for MPC control follows below:

$$
\text { (2) } S_{t+1}=A_{s} S_{t}+B_{s} Q_{t} \text {, }
$$

where $Q_{t}$ is the power flow at the inverter in $\mathrm{kW}, S_{t}$ is the battery state of charge in $\mathrm{kWh}$. In this simulation, the battery rated capacity is $10 \mathrm{kWh}$ and the efficiency is $95 \%$.

\section{3) PV Array}

This unit is supposed to operate at maximum power point (MPP) in order to maximize the revenues from local generation. Therefore, it is assumed that, given the forecast of solar radiance, wind speed and external temperature, the PV controller can forecast the production of the unit via the model-based approach presented in $[3,4]$. In this study, and only for simulation purposes, the PV produces a forecast that is given by the efficient production plus a $\pm 10 \%$ normally distributed error. In this way it is possible to simulate the MPC working under uncertainty conditions.

\section{Control Schemes for the Smart Grid}

The control scheme for HIL simulation is implemented as a hierarchical MPC, as shown in figure 7. Two separate controllers control independently the building heating system and the battery charging cycles. The first controller receives the forecasted PV production, the energy price and the schedule of the building consumption. The latter is computed by the second controller, which strives to minimize the heating costs. Once the consumption schedule is dispatched, the MPC1 controls the relay at the Point of Common Coupling (PCC). In this way, the building is provided with energy either from the islanded system or from the grid. When the building is consuming from the grid, the 


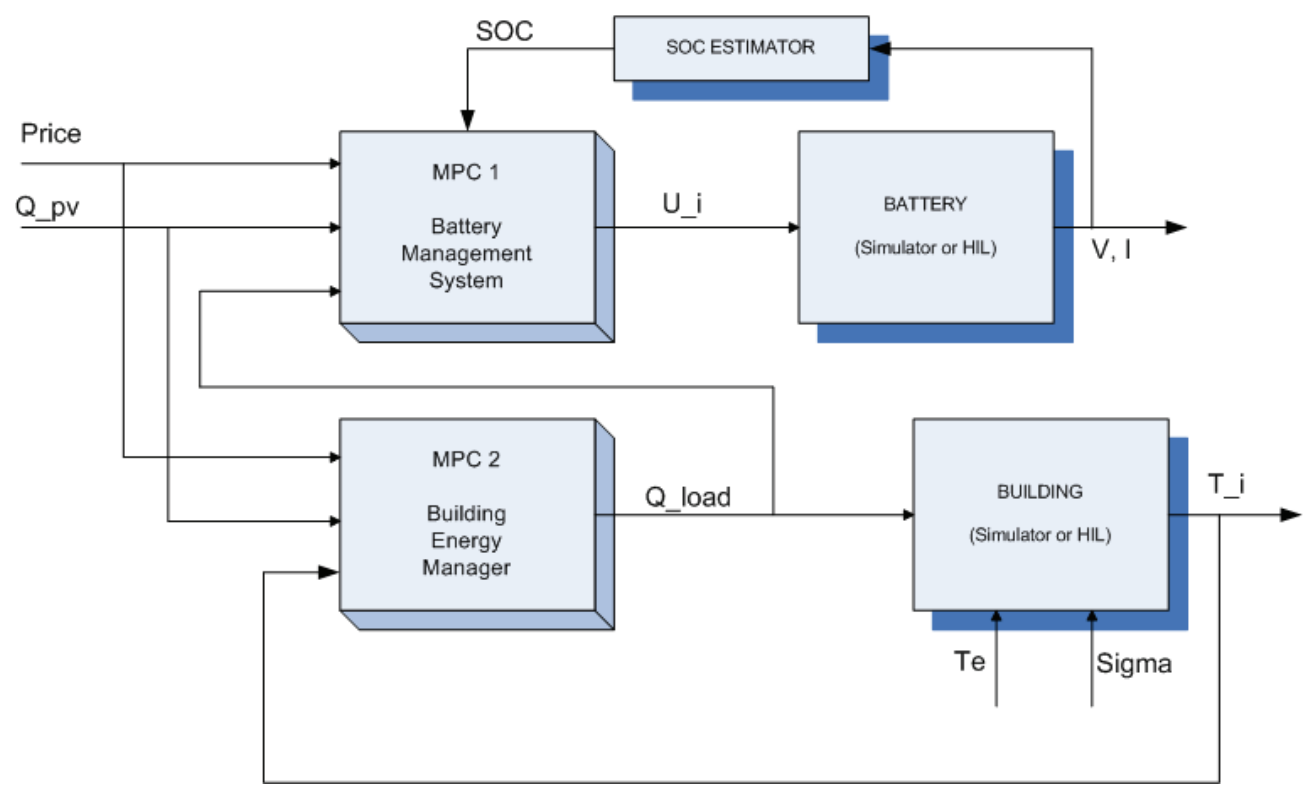

Figure 7. Control architecture

battery is charging. In any case, the PV is connected to the PCC, so that the power produced from a renewable source is automatically directed according to Kirchhoff laws.

1) The MPC objective is to minimize the temperature deviation from the setpoint, the input slew rate and the energy cost:

$$
\begin{array}{cl}
\min _{q_{i}} J=\sum_{i \in T}\left[\left\|T_{i}-T_{r}\right\|_{Q}+\left\|\Delta u_{i}\right\|_{S}+p_{i} q_{i}\right] \\
s . t: & T_{i+1}=A T_{i}+B q_{i}+K T_{e} \\
& u_{\max } \leq u \leq u_{\min } \\
& \Delta u_{\max } \leq \Delta u \leq \Delta u_{\min } \\
\Delta u_{i}=u_{i}-u_{i-1}
\end{array}
$$

The system matrices are obtained by discretization of Equation 1 with a time step of one hour. The control input is constrained between 0 and $300 \mathrm{~W}$, whereas the input slew rate is constrained between $-300 \mathrm{~W}$ and $300 \mathrm{~W}$. The optimization problem defined in Equation 3 is solved via quadratic programming.

2) The battery management system implements an MPC controller with the following formulation:

$$
\begin{array}{rl}
\min _{u_{i}} & H=\sum_{i \in T}\left[u_{i} p_{i}\left(P_{i}^{\text {load }}+P_{i}^{\text {charge }}-P_{i}^{p v}\right)\right] \\
\text { s.t }: & S_{i+1}=A S_{i}+B Q_{i}^{\text {batt }} \\
& Q_{i}^{\text {batt }}=u_{i} P_{i}^{\text {charge }}+\left(1-u_{i}\right)\left(P_{i}^{p v}-P_{i}^{\text {load }}\right) \\
& S_{\min } \leq S_{i} \leq S_{\max } \\
& u_{i} \in\{0,1\}
\end{array}
$$

In Equation $4 P_{i}^{\text {load }}$ is the consumption plan coming from the building heating and cooling management system, $P_{i}^{c h}{ }^{\arg e}$ is the battery charging power and $P_{i}^{p v}$ is the forecasted production plan from the PV unit. The energy price is defined by $p_{i}$ and the control variable for grid connected or islanded mode is $u_{i}$. The profiles for energy price, building consumption and PV production are defined, as well as the battery characteristics (maximum and minimum SOC). The optimization problem defined in Equation 4 is solved via binary integer programming.

In average the problem is solved in less than 4 seconds for a time horizon of 24 hours with a decision granularity of one hour.

\section{Simulation Study}

The simulation study presented in this section shows the efficiency of the hierarchical MPC scheme in managing the local energy storage in order to minimize the energy expenses and maintain the users comfort in the building. Figure 8 regards the building thermal management, where the internal temperature and power consumption for space heating are presented.

The management of the electric storage is shown in figure 9, where the breaker control signal, power flow at the battery and SOC are depicted. Finally, figure 10 shows the Power flow at the PCC and the PV production.

\section{Conclusions}

The present paper discusses the results obtained at the "Measurement, test and control system" (MTCS) in the Power Electronics Laboratory (PEL) of TU, Sofia RDS, Bulgaria. In particular, the paper presents the design, implementation and test of hardware in the loop simulation architecture for integration of Smart Buildings and distributed 

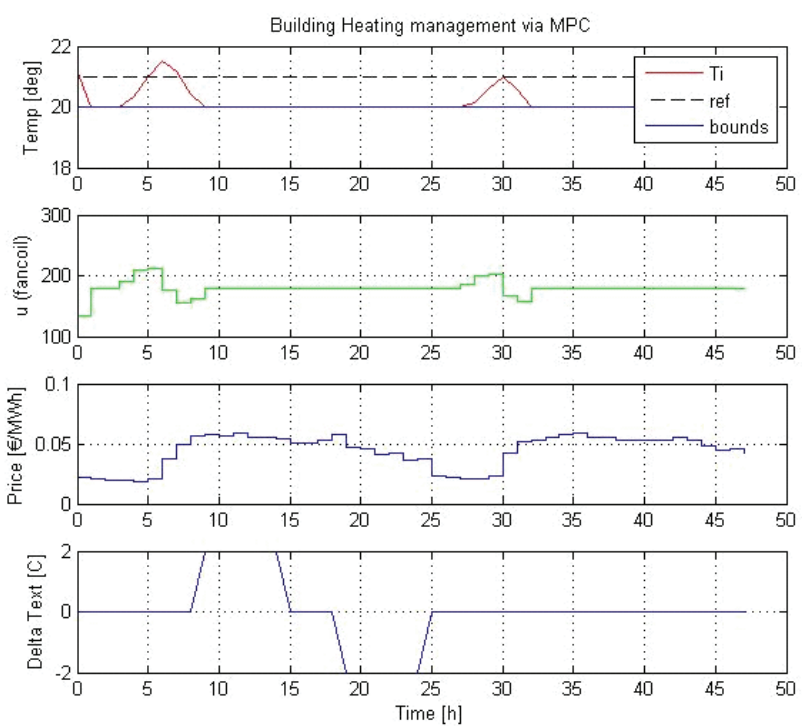

Figure 8. Building space heating management via MPC
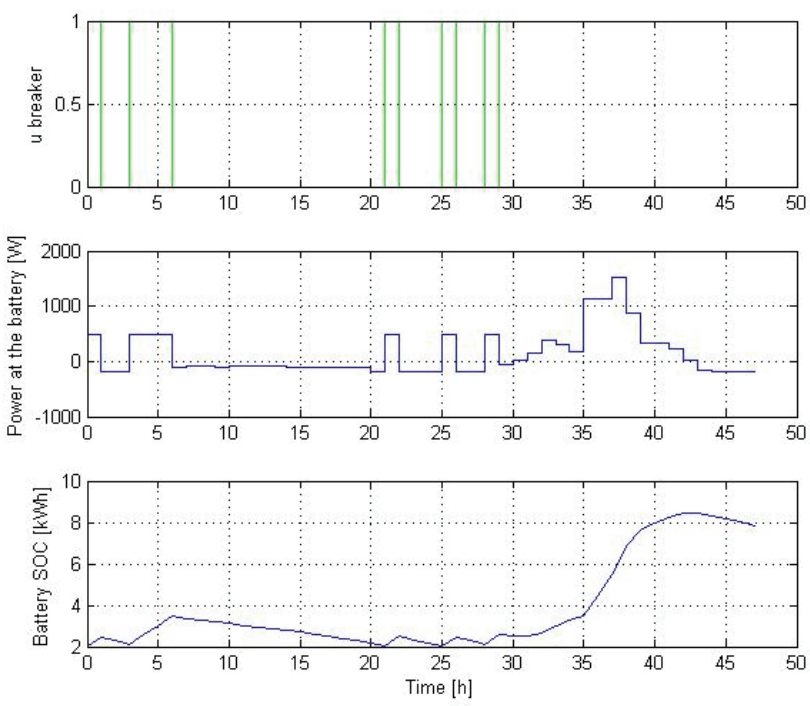

Figure 9. Electric storage management via MPC
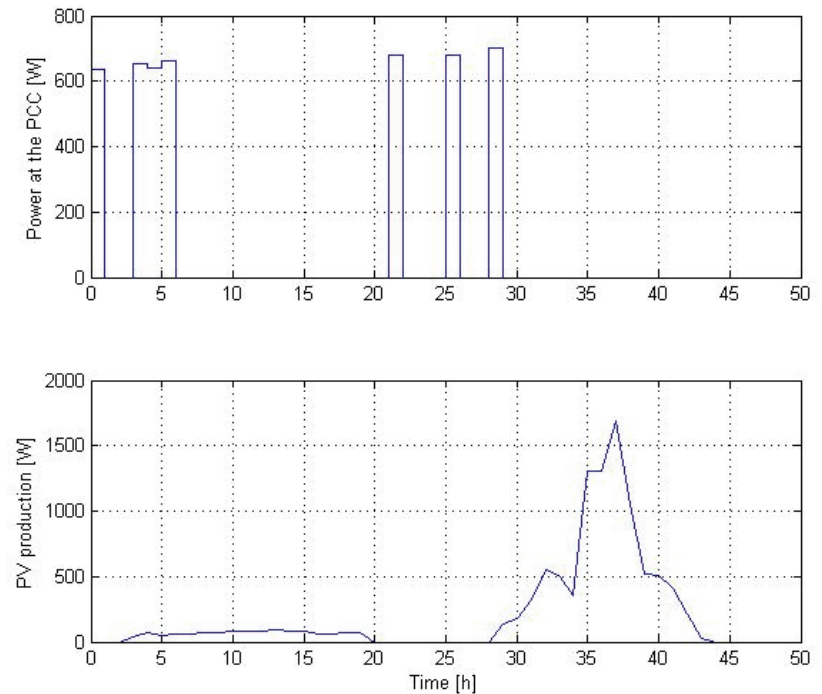

Figure 10. PCC and PV production energy resources in Micro Grids.

The outcomes of the research are:

- Hardware in the loop (HIL) simulation architecture is proposed and validated.

- An innovative method to control a Smart Grid's regimes (on-grid and UPS) based on Building Demand Response is realized.

- Innovative MPC (Model Predictive Control) logic for real-time heating management in buildings is developed.

- Integrated MPC control for energy management in micro grids using batteries and PV under Demand Response Conditions is developed and tested.

- Transnational remote access of a simulation laboratory to hardware facility is demonstrated and tested.

The testing results prove the possibility for remote control of a Smart Grid based on Building Demand Response. The results prove also the feasibility assessment of such a control scheme, and define the aim of integrating the Distributed Generation Sources (DGS) and the storages with residential and commercial customers.

\section{Acknowledgment}

The experimental activities have been supported by the "Transnational Access" program of the European Commission FP7 - Project DERri - Distributed Energy Resources Research Infrastructure - GA No. 228449 see also http://www.der-ri.net.

\section{References}

1. Gellings, C. W. The Concept of Demand-side Management for Electric Utilities. - Proceedings of the IEEE, 73, 1985, No. 10, $1468-1470$

2. Marra, F., G. Y. Yang, C. Traholt, E. Larsen, C. Rasmussen, and S. You. Demand Profile Study of Battery Electric Vehicle under Different Charging Options. Power and Energy Society General Meeting, 2012 IEEE, 2012, 1-7.

3. De Soto, W., S. Klein, and W. Beckman. Improvement and Validation of a Model for Photovoltaic Array Performance. - Solar Energy, 80, 2006, No. 1, 78-88.

4. Koch-Ciobotaru, C., L. Milhet-Popa, F. Isleiffson, and H. Bindner. Simulation Model Developed for a Small-scale PV System in a Distribution Network. Proceedings of the $8^{\text {th }}$ International Symposium of Applied Computational Intelligence and Informatics-SACI 2012, 2012, 257-261.

5. Palensky, P., D. Dietrich. Demand Side Management: Demand Response, Intelligent Energy Systems and Smart Loads. - IEEE Transaction on Industrial Informatics, 7, 2011, 3, 381-388.

6. Romanos, P., N. Hatziargyriou, S. Bonias, N. Daniil, L. Rocca, K. Pantazic, D. Nestle, J. Schmid, P. Strauss, T. Degner, E. Zabala, E. Rodriquez, A. Krusteva, P. Nakov, R. Stanev. Photovoltaics in Smart Buildings. 24th PVSEC, Hamburg, Germany, Proceeding, 2009, 3810-3817.

7. Ferrarini, L., and G. Mantovani. Modeling and Control of Thermal Energy of a Large Commercial Building. Intelligent Energy Systems (IWIES) Vienna, 14 November 2013, IEEE International Workshop on Digital Object Identifier, 10.1109/IWIES.2013.6698577, 149-154. 